\section{Revista Signos}

2010, 43(72)

$31-48$

\title{
Desarrollo fonológico en niños de 3 y 4 años según la fonología natural: Incidencia de la edad y del género
}

\author{
Carmen Julia Coloma \\ María Mercedes Pavez \\ Mariangela Maggiolo \\ Christian Peñaloza \\ Universidad de Chile \\ Chile
}

Resumen: La teoría de la fonología natural plantea que los niños al emitir las palabras las simplifican mediante estrategias denominadas Procesos de Simplificación Fonológica (PSF). Según esta postura, el desarrollo fonológico consiste en la eliminación paulatina de los PSF. Se sabe que entre los 3 y 4 años existe una gran cantidad y variabilidad de subprocesos, sin embargo, no existe una caracterización detallada de este período. Por otra parte, en Chile los estudios sobre el desarrollo fonológico que consideran esta teoría son escasos. Finalmente, la influencia del género ha sido un factor poco estudiado en las investigaciones sobre esta temática. Por ello, se plantean los siguientes objetivos: a) caracterizar el desarrollo fonológico en niños de 3 y 4 años considerando el uso de PSF y sus subprocesos y b) establecer la influencia del género en este desarrollo. Participaron 180 niños organizados en dos grupos etarios, uno de 3 años ( $n$ 90, 42 mujeres y 48 hombres) y otro de 4 años (n 90, 45 mujeres y 45 hombres). Se evaluaron con el TEPROSIF-R que identifica procesos y subprocesos de simplificación fonológica. Se observó que los niños de 3 años efectúan significativamente más PSF que los de 4 años siendo más característicos en ambas edades los subprocesos estructurales. Con respecto al género, se constató que no influía en el desarrollo fonológico. Lo anterior sugiere que los niños y las niñas en este período se centran más en la adquisición de la estructura de la palabra que en las oposiciones fonológicas.

Palabras Clave: Desarrollo fonológico, desarrollo del lenguaje, fonología natural, Procesos de Simplificación Fonológica.

Recibido:

4-IX-2008

Aceptado:

9-VII-2009
Correspondencia: Carmen Julia Coloma (ccoloma@med.uchile.cl). Escuela de Fonoaudiología, Facultad de Medicina, Universidad de Chile. Avenida Independencia 1027, Santiago, Chile. 


\title{
Phonological development in children aged 3 and 4 years considering the use of processes and the influence of gender
}

\begin{abstract}
Natural phonology puts forward that children simplify words in their utterances through strategies known as Phonological Processes (PP). According to this theory, phonological development consists of the gradual fading of PP. Evidence suggests the existence of numerous and diverse PP between 3 and 4 years of age; however, no exact description of this age period is available yet. In Chile, very few phonological development studies consider this theory. In addition, gender influences have been scarcely explored. Therefore, the following objectives were proposed: a) to describe phonological development in 3-4-year-old children by considering their use of PP; and b) to establish gender influences on this development. A sample of 180 participants was organized into two different age groups: a 3-year-old group (n 90; 42 girls and 48 boys) and a 4-year-old group (n 90; 45 girls and 45 boys). Both groups were tested with TEPROSIF-R to identify phonological processes. It was found that 3 year olds use significantly more PP than 4 year olds. Structural PP was more frequent in both groups. Regarding gender, no influence was found for phonological development. Findings suggest that girls and boys in this age period focus more on the acquisition of the structure of words, rather than on phonological oppositions.
\end{abstract}

Key Words: Phonological development, language development, natural phonology, Phonological Processes.

\section{INTRODUCCIÓN}

Estudiar el desarrollo fonológico es una tarea que requiere considerar las discrepancias entre las producciones lingüísticas de los adultos y las de los niños, establecer los patrones del desarrollo fonológico infantil y determinar la unidad básica (fonema o palabra) que se considerará (Galeote, 2002; Acosta, León \& Ramos, 1998).

Por ello no es sorprendente que se hayan utilizado diversas perspectivas para abordar el tema. Algunas se centran en la secuencia de aparición de fonemas y de los rasgos que los caracterizan (teorías conductistas y estructuralistas). Otras, como la fonología natural, se focalizan en la adquisición de la fonología de la palabra. Esta última propuesta resulta muy útil y económica para describir la relación entre la producción adulta y la infantil (Dodd, Holm, Hua \& Crosbie, 2003). Plantea que el niño escucha la palabra adulta y la reproduce simplificada fonológicamente, para lo cual aplica estrategias conocidas como procesos de simplificación fonológica (PSF). Dichas estrategias son operaciones mentales que constituyen un sistema innato. Se distinguen tres tipos de PSF: relacionados con la estructura de la sílaba y de la palabra, de sustitución y de asimilación (Stampe, 1969; Ingram, 1983). Cada uno de estos tipos de PSF se manifiesta en subprocesos específicos. 
En los procesos relacionados con la estructura de la sílaba y de la palabra, se simplifican las emisiones acercándolas a la estructura silábica básica (consonante + vocal). Para ello, por ejemplo, se suprimen codas (/elikóptero/-/elikó_tero/) o se reducen grupos consonánticos (/ tren/-/ten/), fenómenos que corresponden a los subprocesos de omisión de consonante trabante y reducción de grupo consonántico respectivamente. También se simplifica la palabra a través de la modificación de su metría, como es el caso de la omisión de sílabas átonas (/ maripósa/-/_pósa/), subproceso denominado omisión de elementos átonos.

Los procesos de asimilación son estrategias mediante las cuales se reemplaza un fonema para hacerlo igual o semejante a otro presente en la palabra. La similitud entre fonemas se produce fundamentalmente por zona de articulación. Un ejemplo de este tipo de asimilaciones es el subproceso de asimilación velar (/bufánda/-/gufánda/). Por su parte, la asimilación idéntica es un subproceso en que un fonema se hace igual a otro (/kaperusíta/-/kaperutíta/).

Por último, en los procesos de sustitución se cambian fonemas pertenecientes a una clase por miembros de otra clase fonemática, por ejemplo, el subproceso de oclusivización de fonemas fricativos en que se sustituyen fricativos por oclusivos (/puénte/-/fuénte/) (Pavez, Maggiolo \& Coloma, 2008).

La teoría de la fonología natural plantea que el niño posee una representación fonológica de la palabra igual a la del adulto, aunque la produzca con errores. Al respecto, se ha discutido la realidad psicológica y el poder explicativo de este postulado (Dodd et al., 2003). Por ello, otra perspectiva sobre los PSF ha propuesto que operan sobre las representaciones de las palabras, facilitando la producción lingüística del niño (Martínez, Díez-Itza \& Miranda, 2004). Aun cuando la fonología natural ha sido criticada, se reconoce que es útil para comprender las dificultades fonológicas infantiles y para elaborar programas de intervención que permitan superarlas (Galeote, 2002).

Se ha especificado que las simplificaciones pueden realizarse en la palabra, en la sílaba o en el fonema. Así, a nivel de la palabra se encontrarían las asimilaciones y las modificaciones de la metría. En cambio, la reducción del grupo consonántico y la omisión de coda son procesos de la sílaba, mientras que las sustituciones corresponden al plano del fonema (Serra, Serrat, Solé, Bel \& Aparici, 2000).

Otra propuesta que se ha efectuado en cuanto a los PSF es la de clasificarlos en estructurales y sistémicos. Los primeros incluyen los procesos de asimilación y los relacionados con la estructura de la sílaba y de la palabra. Los sistémicos, por su parte, se centran en las oposiciones fonológicas y corresponden a los de sustitución (Bosch, 2003, 2004). Esta distinción es útil ya que contribuye a precisar el tipo de dificultades fonológicas que presentan algunos niños y 
posibilita planificar las estrategias de intervención más eficaces para ellos. Lo anterior, porque desde la perspectiva clínica, se reconoce que los niños con mayor presencia de PSF estructurales manifiestan problemas fonológicos más severos que aquellos que evidencian principalmente PSF sistémicos.

Según la fonología natural, el desarrollo fonológico implica la progresiva eliminación de los PSF hasta que el niño logra producir la palabra como la emite el adulto. Por cierto, la disminución de PSF coexiste con la adquisición del sistema de fonemas.

Al respecto se ha comprobado que el mayor número de procesos y subprocesos se observa a los 3 y 4 años en niños hablantes del inglés (Dodd et al., 2003); hecho que también se ha constatado en niños hispanohablantes (Bosch, 2004). Además, se ha establecido que en estas edades los hablantes del español presentan gran variabilidad en sus rendimientos al compararlos con menores de 5 años (Martínez et al., 2004).

Además, se han caracterizado patrones fonológicos considerando los PSF en niños descendientes de puertorriqueños de 3 y 4 años, residentes en Estados Unidos, cuya lengua materna es el español. Centrándose en la cantidad de subprocesos utilizados, se encontró que en los dos grupos etarios la mayoría de ellos no superaba el $10 \%$ de frecuencia en relación al total de procesos producidos por los niños, es decir, existía una gran variabilidad en sus desempeños (Goldstein \& Iglesias, 1996a).

En síntesis, se advierte que entre los 3 y 4 años los PSF son abundantes y sus subprocesos específicos son diversos.

Por otra parte, en este mismo período se produce un importante desarrollo fonológico donde se enriquece el conocimiento sobre la estructura fonológica de las palabras y sobre el sistema fonemático de la lengua, fenómeno que se relaciona de modo relevante con el incremento significativo del léxico (Bosch, 2003). Existen, sin embargo, menores que no logran desarrollar adecuadamente su fonología. Ello se evidencia en un mayor uso de PSF y de sus subprocesos, lo que implica que su lenguaje aparece ininteligible y similar al de un niño más pequeño. Con el fin de contribuir a detectar a estos sujetos, es necesario contar con una caracterización adecuada y pormenorizada de los procesos y, en especial, de los subprocesos que son usados por niños sin problemas en su desarrollo lingüístico.

Como se señaló previamente, entre los 3 y 4 años el uso de PSF es abundante y los subprocesos muy variables. Un factor que puede influir en esta variabilidad, además de las diferencias individuales, es el género. Al respecto, cuando se aborda la posible relación entre adquisición del lenguaje y género, suele atribuirse a las niñas superioridad en el desarrollo lingüístico señalan- 
do que comienzan a hablar más temprano (Garayzábal, 2006; Murray, Johnson \& Peters, 1990); adquieren más rápido el vocabulario (Roulstones, Loader \& Northstone, 2002) y se expresan espontáneamente con más frecuencia (Bauer, Goldfield \& Reznick, 2002). Se ha encontrado también una ventaja significativa en la edad de adquisición de las primeras 50 palabras en las mujeres (D'Odorico, Carubbi, Salerni \& Calvo, 2001), hecho que no ha sido corroborado en otras investigaciones en que niños y niñas presentan desempeños similares (Bassano, Maillonson \& Eme, 1998).

Trabajos más recientes abordan las diferencias de género intentando determinar principalmente su influencia en el procesamiento neurológico del lenguaje en la niñez. Así, algunos hallazgos, basados en técnicas de neuroimagen en las que se presentan palabras de modo visual y auditivo, sugieren que en las niñas existe una red supramodal en el procesamiento neural del lenguaje. Los varones, en cambio, procesan de manera diferente las palabras según la modalidad utilizada. Ello se relaciona con que en el cerebro de las niñas se observa una mayor activación bilateral en áreas frontales inferiores, en el giro temporal superior y el giro fusiforme izquierdo en determinadas tareas lingüísticas (Burman, Bitan \& Booth, 2008).

En el estudio del desarrollo fonológico, el género es un factor que se ha investigado, aunque su posible influencia no ha sido suficientemente consensuada. Al respecto, en investigaciones con niños de habla inglesa se ha observado que a los 3 y 4 años ambos géneros manifiestan un comportamiento fonológico similar, por el contrario, entre los 5 y 6 años existe mayor precisión en la producción de fonemas en las niñas (Dodd et al., 2003). En hablantes de lengua española, al estudiar subprocesos muy específicos como la metátesis desde los 3 hasta los 5 años 6 meses, se ha advertido que su frecuencia de aparición es constante en las niñas mientras que en los varones decrece de modo uniforme hasta los 4 años 6 meses y posteriormente se hace irregular (Díez-Itza \& Martínez, 2003). Sin embargo, al analizar la producción de fonemas en distintos contextos a partir de narraciones infantiles en niños y niñas hispanohablantes de 3 a 5 años, no se han constatado diferencias significativas en cuanto al género (Dioses, García, Matalinares, Cuzcano, Panca, Quiroz, Fernández \& Castillo, 2006).

En el ámbito de la patología del lenguaje, el trastorno fonológico es frecuente y existe evidencia sobre la importancia del género, puesto que la cantidad de niños que presentan problemas es mayor que el número de niñas. No obstante, los déficits de estas últimas suelen ser más severos que los de los varones (Law, 1992). Por ello, es necesario considerar el género en el diagnóstico y en el tratamiento de los problemas del lenguaje.

Según lo expuesto hasta ahora, precisar los subprocesos y la influencia del género en niños de 3 y 4 años enriquecería la comprensión del desarrollo fonológico en hispanohablantes y permitiría 
contar con una descripción más pormenorizada de los subprocesos. Lo anterior puede resultar particularmente útil para identificar niños con problemas fonológicos.

De este modo, los objetivos de este trabajo son: a) caracterizar el desarrollo fonológico en niños de 3 y 4 años considerando el uso de PSF y sus subprocesos; y b) establecer la influencia del género en el desarrollo fonológico de los niños en esas edades.

\section{Metodología}

\subsection{Participantes}

Se trabajó con 180 niños divididos en dos grupos etarios. El primero está constituido por 90 sujetos de 3.0 a 3.11 años (42 mujeres y 48 hombres) y el segundo, por 90 niños de 4.0 a 4.11 años (45 mujeres y 45 hombres). Todos eran alumnos de jardines infantiles y evidenciaban un desarrollo lingüístico apropiado para su edad. Esto último se constató en el informe escolar, a través del cual se corroboró además que no asistían a escuelas de lenguaje ni a tratamiento fonoaudiológico.

El nivel socioeconómico se controló, ya que en cada grupo, 30 participantes pertenecían al nivel medio bajo, 30 al nivel medio medio y 30 al nivel medio alto. Se seleccionaron esos tres niveles porque en ellos se agrupa la mayoría de los hogares de Chile según ADIMARK (2004). Para establecer el nivel socioeconómico se consideró la educación, la profesión y la actividad del jefe de hogar según los criterios de ICCOM (2005).

\subsection{Procedimientos}

Se evaluó el desempeño de los niños con el Test para Evaluar Procesos de Simplificación Fonológica, edición revisada, TEPROSIF-R (Pavez et al., 2008). Dicha prueba es la única en Chile que evalúa PSF, posee normas y estudios de validez. Además, cuenta con un alto índice de confiabilidad (coeficiente Alfa Cronbach: 0.90).

Es importante señalar que las normas que ofrece este instrumento están referidas a las tres grandes categorías de PSF (relacionados con la estructura de la sílaba y de la palabra, asimilación y sustitución). Por lo tanto, la prueba no contempla referencias a los subprocesos de las categorías señaladas. Sin embargo, presenta definiciones y criterios para analizarlos, los que fueron utilizados en esta investigación.

El TEPROSIF-R consta de 37 ítemes correspondientes a palabras de distinta metría, acentuación 
y complejidad silábica. Así, se incluyen monosílabos, bisílabos, trisílabos y polisílabos, palabras graves, agudas y esdrújulas con sílabas de diferente estructura (CV, VV, CVC, CCV, CCVC, (VVC).

Para elicitar las palabras infantiles se utiliza la imitación diferida con apoyo de un set de láminas. Las dos primeras se emplean como ejemplos con el fin de familiarizar al niño con la tarea. La instrucción consiste en señalar un dibujo en la parte superior de la lámina diciendo al menor una oración que finaliza con la palabra estímulo (Mira, aquí hay una mariposa), luego indicando en la parte inferior otro dibujo se enuncia una oración donde falta al final el estímulo evaluado y se le pide completarla (y ahora mira acá, en las flores está la...).

Los niños fueron evaluados de forma individual en una sala de clases, silenciosa y sin distractores. Sus respuestas se transcribieron fonológicamente y luego, según los criterios del TEPROSIF-R, se identificaron los PSF y sus subprocesos en cada una de las palabras emitidas por ellos. Para tal efecto, se usó la hoja de análisis del test. En ella se presentan las 37 palabras de la prueba divididas en sílabas y se consigna en cursiva la sílaba acentuada. Debajo de cada término se escriben fonológicamente las respuestas del menor y se analizan los PSF y sus subprocesos, según los criterios de corrección del test.

Se inició el análisis identificando las tres grandes categorías de procesos. Para ello, se utilizó la secuencia propuesta por la prueba según la cual primero se identifican los procesos relacionados con la estructura de la sílaba y de la palabra, luego los de asimilación y finalmente los de sustitución. Una vez establecidas estas tres grandes categorías se consignaron los subprocesos de cada una de ellas. Este análisis se efectuó, como se mencionó previamente, basándose en las definiciones del TEPROSIF-R. Así por ejemplo, si un niño dice /bolatín/ en vez de /bolantín/ en primer término se establece que es un proceso relacionado con la estructura de la sílaba y de la palabra y posteriormente se identifica el subproceso específico que en este caso corresponde a omisión de consonante trabante o de coda silábica.

A continuación, se detallan las definiciones de aquellos subprocesos que resultaron más frecuentes en este estudio.

\section{Subprocesos relacionados con la estructura de la sílaba y de la palabra}

Omisión de elementos átonos: Se eliminan sílabas átonas o alguno de los fonemas que las constituyen. Ejemplo: /maripósa/ /ma_pósa/. Cuando se omiten elementos contiguos aunque pertenezcan a sílabas diferentes (/ efrixeradór/ / efrix_adór/) o corresponden a varias sílabas (/edifísio//_físio/) se consigna como un solo subproceso. En cambio, si los elementos omiti- 
dos no son contiguos, cada omisión es considerada como un subproceso (/ efrixeradór/ /_ fri_adór/). Ello se debe a que se contabiliza el número de veces que el niño usa la estrategia y no su efecto en la palabra simplificada.

Omisión de consonante trabante o coda silábica: Se omite el fonema consonántico ubicado al final de la sílaba. Ejemplo: /pantalón/ /pa_talón/.

Reducción de grupo consonántico: Se omite uno de los fonemas del grupo consonántico. Ejemplo: /plátano/ /p_átano/.

Reducción de diptongo: Se elimina uno de los fonemas del diptongo que es la secuencia de una vocal abierta tónica + una vocal cerrada átona o viceversa. Ejemplo: /puénte/ /p_énte/.

\section{Subprocesos de Asimilación}

Asimilación idéntica: Un fonema se cambia para hacerse idéntico a otro fonema presente en la palabra. Ejemplo: /bisikléta/ /bisikléka/.

Asimilación velar: Un fonema se hace similar a uno velar consonántico (/k/, /g/, /x/) o vocálico como /o/o /u/. Ejemplo: / uéda//guéda/.

\section{Subprocesos de Sustitución}

Sustitución de fonemas líquidos entre sí: Se sustituye un fonema líquido por otro líquido.

Ejemplo: /gó o/ /góro/.

Sustitución de líquidos por no líquidos orales: Se sustituye un fonema líquido (/r/, / / , ///) por un fonema no líquido oral. Ejemplo: / elóx/ /delóx/.

Previo al análisis de las respuestas, se constató la confiabilidad interexaminadores con el coeficiente de concordancia W de Kendall. Al tercer intento, se logró un índice de 0.95 (p 0.000 ) considerando el total de PSF identificados en el test. Similar procedimiento se utilizó al analizar los tres tipos de procesos y subprocesos obteniéndose un índice de 0.96 ( $p$ 0.000) para PSF relacionados con la sílaba, de 0.91 (p 0.000) para los de asimilación y de 0.84 en los de sustitución ( $p$ 0.000). 


\section{Resultados}

Primero se realizó un análisis descriptivo donde se advirtió que el grupo de niños de 3 años emitió un total de 2.394 PSF, con un mínimo de 1 subproceso por niño y un máximo de 68. El grupo de 4 años, en cambio, elicitó 1.186 PSF con un mínimo de 0 y un máximo de 50 . Lo anterior constata la mayor frecuencia de PSF y sus subprocesos en los niños de 3 años.

Para verificar si la diferencia entre los rangos etarios era estadísticamente significativa, se compararon mediante una t de Student sus promedios en el total de PSF y en cada tipo de ellos. Los resultados se aprecian en la Tabla 1. Además, se calculó el tamaño del efecto en las comparaciones y se advirtió que en el total de PSF su valor fue 1.11; en los PSF relacionados con la sílaba y la palabra, 1.0; en asimilación, 0.95 y en sustitución, 0.86 . Es decir, la diferencia entre los grupos es fuerte.

Tabla 1. Comparación del promedio del total de PSF y de cada tipo en niños de 3 y 4 años.

\begin{tabular}{|c|c|c|c|c|c|}
\hline $\begin{array}{l}\text { Grupos etarios } \\
\text { Procesos de } \\
\text { Simplificación Fonológica }\end{array}$ & \multicolumn{2}{|c|}{$\begin{array}{c}\text { Niños de } \\
3 \text { años (n 90) }\end{array}$} & \multicolumn{2}{|c|}{$\begin{array}{c}\text { Niños de } \\
4 \text { años (n 90) }\end{array}$} & t de Student \\
\hline Total de PSF & 26.60 & 14.34 & 13.17 & 9.39 & $7.42^{* *}$ \\
\hline \multicolumn{6}{|l|}{ Tipos de PSF } \\
\hline $\begin{array}{l}\text { Relacionados con la estructura } \\
\text { de la sílaba y de la palabra }\end{array}$ & 13.98 & 7.96 & 7.10 & 5.96 & $6.68^{* *}$ \\
\hline Asimilación & 6.77 & 4.80 & 3.04 & 2.75 & $6.39^{* *}$ \\
\hline Sustitución & 5.83 & 3.79 & 3.03 & 2.65 & $5.73^{* *}$ \\
\hline
\end{tabular}

** p 0.000 PROM: promedio; D.E.: Desviación estándar.

Los resultados en la Tabla 1 indican que los niños de 4 años efectúan significativamente menos PSF que los de 3, tanto en el total de procesos como en cada tipo de ellos. Se advierte también al observar la desviación estándar la gran variabilidad en el desempeño de los niños de ambos grupos etáreos.

Luego, para establecer los subprocesos más característicos a los 3 y a los 4 años, se calculó el porcentaje de niños que los utilizaba en cada grupo etario. Se consideraron los subprocesos particulares pertenecientes a los tres grandes tipos de PSF y ocho rangos de porcentaje (de menos del $10 \%$ de sujetos hasta el $90 \%$ o más). Los resultados se presentan en la Tabla 2. 
Tabla 2. Porcentaje de niños que utiliza los distintos subprocesos en cada grupo etario.

\begin{tabular}{|c|c|c|c|c|}
\hline $\begin{array}{l}\text { Rangos según } \\
\text { porcentaje } \\
\text { de niños }\end{array}$ & \multicolumn{2}{|l|}{ Niños de 3 años } & \multicolumn{2}{|l|}{ Niños de 4 años } \\
\hline $90 \%$ o más & 1. Omisión de elementos átonos & 97.8 & & \\
\hline $\begin{array}{c}\text { entre } \\
70 \% \text { y } 89 \%\end{array}$ & $\begin{array}{l}\text { 2. Asimilación idéntica } \\
\text { 3. Omisión de trabante } \\
\text { 4. Líquidos entre sí }\end{array}$ & $\begin{array}{l}88.9 \% \\
81.1 \% \\
73.3 \%\end{array}$ & $\begin{array}{l}\text { 1. Omisión de elementos átonos } \\
\text { 2. Asimilación idéntica } \\
\text { 3. Omisión de trabante }\end{array}$ & $\begin{array}{l}86.7 \% \\
77.8 \% \\
70.0 \%\end{array}$ \\
\hline $\begin{array}{c}\text { entre } \\
50 \% \text { y } 69 \%\end{array}$ & $\begin{array}{l}\text { 5. Reducción de grupo consonántico } \\
\text { 6. Reducción de grupo vocálico }\end{array}$ & $\begin{array}{l}66.7 \% \\
57.8 \%\end{array}$ & 4. Reducción de grupo consonántico & $56.7 \%$ \\
\hline $\begin{array}{c}\text { entre } \\
40 \% \text { y } 49 \%\end{array}$ & $\begin{array}{l}\text { 7. Aspiración de trabantes } \\
\text { 8. Asimilación velar } \\
\text { 9. Adición de fonemas o sílabas } \\
\text { 10. Líquidos por no líquidos }\end{array}$ & $\begin{array}{l}48,9 \% \\
46.7 \% \\
44.4 \% \\
41.1 \% \\
\end{array}$ & 5. Líquidos entre sí & $44.4 \%$ \\
\hline $\begin{array}{l}\text { entre } \\
30 \% \text { y } 39 \%\end{array}$ & $\begin{array}{l}\text { 11. Metátesis } \\
\text { 12. Asimilación dental } \\
\text { 13. Coalescencia } \\
\text { 14. Frontalización } \\
\end{array}$ & $\begin{array}{l}35.6 \% \\
34.4 \% \\
33.3 \% \\
31.1 \% \\
\end{array}$ & 6. Aspiración de trabantes & $34.4 \%$ \\
\hline $\begin{array}{l}\text { entre } \\
20 \% \text { y } 29 \%\end{array}$ & $\begin{array}{l}\text { 15. Oclusivización } \\
\text { 16. Asimilación nasal } \\
\text { 17. Asimilación palatal } \\
\text { 18. No líquidos por líquidos }\end{array}$ & $\begin{array}{l}27.8 \% \\
24.4 \% \\
22.2 \% \\
20.0 \% \\
\end{array}$ & $\begin{array}{l}\text { 7. Oclusivización } \\
\text { 8. Asimilación velar }\end{array}$ & $\begin{array}{l}23.3 \% \\
22.2 \%\end{array}$ \\
\hline $\begin{array}{c}\text { entre } \\
10 \% \text { y } 19 \%\end{array}$ & $\begin{array}{l}\text { 19. Semiconsonantización de líquidos } \\
\text { 20. Asimilación vocálica } \\
\text { 21. Asimilación a líquidos } \\
\text { 22. Fricativización } \\
\text { 23. Omisión de elementos tónicos } \\
\text { 24. Labialización } \\
\text { 25. Pérdida de sonoridad } \\
\text { 26. Asimilación silábica } \\
\text { 27. Disimilación }\end{array}$ & $\begin{array}{l}17.8 \% \\
14.5 \% \\
14.5 \% \\
13.3 \% \\
12.2 \% \\
12.2 \% \\
12.2 \% \\
10.0 \% \\
10.0 \% \\
\end{array}$ & $\begin{array}{l}\text { 9. Asimilación dental } \\
\text { 10. Frontalización } \\
\text { 11. No líquidos por líquidos } \\
\text { 12. Líquidos por no líquidos } \\
\text { 13. Asimilación palatal }\end{array}$ & $\begin{array}{l}17.8 \% \\
15.6 \% \\
13.3 \% \\
11.1 \% \\
10.0 \%\end{array}$ \\
\hline$<10 \%$ & $\begin{array}{l}\text { 28. Posteriorización } \\
\text { 29. Nasalización } \\
\text { 30. Oralización de nasales }\end{array}$ & $\begin{array}{l}6.7 \% \\
3.4 \% \\
1.1 \%\end{array}$ & $\begin{array}{l}\text { 14. Semiconsonantización de líquidos } \\
\text { 15. Pérdida de sonoridad } \\
\text { 16. Asimilación Vocálica } \\
\text { 17. Fricativización } \\
\text { 18. Disimilación } \\
\text { 19. Asimilación a líquidos } \\
\text { 20. Nasalización } \\
\text { 21. Omisión de elementos tónicos } \\
\text { 22. Oralización de nasales } \\
\text { 23. Asimilación silábica } \\
\text { 24. Posteriorización }\end{array}$ & $\begin{array}{l}7.8 \% \\
6.7 \% \\
5,6 \% \\
5.6 \% \\
5.6 \% \\
4,4 \% \\
4.4 \% \\
3,3 \% \\
3.3 \% \\
2,2 \% \\
2.2 \%\end{array}$ \\
\hline
\end{tabular}


Se advierte en la Tabla 2 que los niños de ambas edades realizan una gran diversidad de subprocesos (30 a los 3 años y 24 a los 4). Sin embargo, la mayoría son utilizados por menos del 50\% de los participantes.

Los subprocesos más frecuentes en ambas edades son omisión de elementos átonos, asimilación idéntica y omisión de trabante. Además, el 73.3\% de los menores de 3 años efectúa sustitución de líquidos entre sí, lo que ocurre solo en un $44.4 \%$ en los de 4 años.

Otro hecho destacable es que entre el $50 \%$ y el $69 \%$ de los niños de ambas edades está presente el subproceso reducción de grupo consonántico, mientras que la reducción de grupo vocálico se advierte solo a los 3 años en este rango de porcentajes.

Finalmente, para establecer la importancia del género en el desarrollo fonológico se comparó, mediante una t de Student, el uso de PSF entre niños y niñas considerando el total de procesos y cada tipo de ellos. Los resultados obtenidos se observan en la Tabla 3.

Tabla 3. Comparación del promedio del total de PSF y de cada tipo entre ambos géneros en niños de 3 y 4 años.

\begin{tabular}{|c|c|c|c|c|c|c|c|c|c|c|}
\hline \multirow{3}{*}{$\begin{array}{l}\text { Grupos según } \\
\text { edad y género } \\
\text { Procesos de } \\
\text { Simplificación } \\
\text { Fonológica }\end{array}$} & \multicolumn{5}{|c|}{3 años } & \multicolumn{5}{|c|}{4 años } \\
\hline & \multicolumn{2}{|c|}{$\begin{array}{l}\text { Niñas } \\
\text { (n 42) }\end{array}$} & \multicolumn{2}{|c|}{$\begin{array}{l}\text { Niños } \\
\text { (n 48) }\end{array}$} & \multirow[b]{2}{*}{$\mathrm{t}$} & \multicolumn{2}{|c|}{$\begin{array}{l}\text { Niñas } \\
\text { (n 45) }\end{array}$} & \multicolumn{2}{|c|}{$\begin{array}{l}\text { Niños } \\
\text { (n 45) }\end{array}$} & \multirow[b]{2}{*}{$\mathrm{t}$} \\
\hline & PROM. & D.E. & PROM. & D.E. & & PROM. & D.E. & PROM. & D.E. & \\
\hline Total de PSF & 26.1 & 15.5 & 27.0 & 13.4 & 0.2 N.S. & 12.2 & 7.5 & 14.1 & 10.9 & 0.9 N.S. \\
\hline \multicolumn{11}{|l|}{ Tipos de PSF } \\
\hline $\begin{array}{l}\text { Procesos } \\
\text { relacionados } \\
\text { con la sílaba }\end{array}$ & 14.7 & 8.7 & 13.8 & 7.3 & 0.1 N.S. & 6.5 & 4.4 & 7.7 & 6.7 & 1.0 N.S. \\
\hline $\begin{array}{l}\text { Procesos de } \\
\text { Asimilación }\end{array}$ & 6.1 & 4.8 & 7.3 & 4.7 & 1.1 N.S. & 2.9 & 2.6 & 3.2 & 2.9 & 0.5 N.S. \\
\hline $\begin{array}{l}\text { Procesos de } \\
\text { Sustitución }\end{array}$ & 5.9 & 4.2 & 5.8 & 3.4 & 0.1 N.S. & 2.8 & 2.2 & 3.3 & 3.0 & 0.9 N.S. \\
\hline
\end{tabular}

N.S.: No significativo; PROM: promedio; D.E.: Desviación estándar.

La Tabla 3 muestra que no existen diferencias significativas entre varones y niñas en el total de PSF ni en sus tres tipos. En consecuencia, esta variable no influye en el manejo de procesos en los participantes estudiados. 


\section{Discusión final}

Tal como lo plantea la fonología natural, los resultados de esta investigación corroboran la disminución significativa de PSF y de sus subprocesos en el tramo de edad estudiado. Ello concuerda con trabajos previos en los que se ha evidenciado que los niños de 3 años efectúan mayor número de estas estrategias fonológicas que los de 4 años (Martínez et al., 2004; Storti, 2002; Serra, et al., 2000; Bosch, 2004). También, se ratifica la variabilidad de los rendimientos, como ya se ha descrito en otros estudios (Martínez, et al., 2004).

Con respecto al desempeño característico, se observa que en ambos grupos etarios la mayor cantidad de subprocesos son estructurales (relacionados con la estructura de la sílaba y de la palabra y asimilaciones). Este hallazgo coincide con lo encontrado en un estudio realizado con niños entre 2 y 5 años, donde los subprocesos más comunes son de tipo estructural (Storti, 2002).

En el presente estudio, los subprocesos estructurales más frecuentes en ambas edades fueron la omisión de elementos átonos y la asimilación idéntica, seguidos por la omisión de trabante.

Es posible asociar la frecuencia de la omisión de elementos con la tendencia en los niños a mantener las unidades tónicas en la palabra, ya que son las más perceptibles y estables. En consecuencia, un factor que puede influir en las simplificaciones de estas edades es el grado de perceptibilidad de los elementos constitutivos de la palabra. El hecho de que menores de 2 años con desarrollo típico de lenguaje omitan la sílaba átona inicial, al igual que niños mayores con Trastorno Específico del Lenguaje, corrobora que las sílabas átonas son más difíciles de percibir y producir que las tónicas (Carter \& Gerken, 2003).

Por su parte, la asimilación idéntica incluye todas las asimilaciones donde un fonema se iguala a otro presente en la palabra. Este subproceso fue propuesto en el TEPROSIF- R para diferenciarlo de aquellos donde el fonema solo se asimila a otro (Pavez, et al., 2008). Cuando el niño simplifica con asimilaciones, armoniza los fonemas que constituyen una palabra, lo que se logra más aún con las asimilaciones idénticas. Así, la frecuencia de ellas sugiere que los menores requieren de un alto grado de armonización para lograr producir la palabra.

Otros subprocesos frecuentes en esta investigación fueron la omisión de consonante trabante y la reducción de grupo consonántico. Esta última estrategia parece característica del desarrollo fonológico en hablantes del español con desarrollo típico del lenguaje (Bosch, 2004) y también en niños de 3 y 4 años con problemas fonológicos (Goldstein \& Iglesias, 1996b).

El predominio de ambos subprocesos se relaciona con los diferentes tipos de estructuras silábicas en los cuales incide la presencia o ausencia de sus elementos constituyentes. La sílaba consta de un núcleo vocálico y dos unidades opcionales: el ataque y la coda. A su vez, el ataque 
está conformado por al menos un fonema que se sitúa antes del núcleo mientras que la coda corresponde a uno o más fonemas ubicados después del núcleo (Belinchón, Igoa \& Rivière, 2000). Según la cantidad de fonemas que componen estas unidades, se distinguen ataques y codas simples o complejos. Así, son simples cuando están constituidos por un fonema y son complejos cuando están conformados por un grupo de ellos.

La omisión de consonante trabante implica la eliminación de la coda y la reducción de grupo consonántico requiere de la simplificación de un ataque complejo. Mediante dichos subprocesos el niño emite una sílaba básica que posee consonante (ataque simple) + vocal (núcleo) (D’Introno, Del Teso \& Weston, 1995) que es la más común en español (Navarro Tomás, 1966) y que es considerada como la más fácil de producir (Clemente, 1996). En consecuencia, la utilización de estos subprocesos sugiere que el menor para emitir la palabra necesita simplificar la estructura silábica con el fin de producir sílabas básicas.

También la mayor frecuencia de ambos subprocesos se asocia con la adquisición de fonemas. Así, un fonema aparece inicialmente en posición intervocálica y luego su uso se generaliza secuencialmente en posición inicial absoluta de palabra, posición final absoluta de palabra, coda en interior de palabra y, por último, en ataque complejo (Oropeza, 2001). Por lo tanto, el manejo de la coda y del grupo consonántico se logra más tardíamente y es probable que por ello sea común su simplificación a los 3 y 4 años.

Por otra parte, aunque la metátesis no es frecuente, es interesante su análisis porque ha sido caracterizada como un subproceso atípico que manifiesta una gran variabilidad inter e intrasujetos y no se relaciona con la edad, con el número total de subprocesos ni con el vocabulario (Díez-Itza \& Martínez, 2003). En la presente investigación, la metátesis aparece solo a los 3 años en un $35.6 \%$ de los niños y a los 4 años no se observa. Lo anterior no concuerda con lo constatado en otra investigación realizada con menores de 3 a 6 años donde se observó que no desaparece con la edad (Díez-Itza \& Martínez, 2003). Es posible que las distintas modalidades utilizadas en las producciones fonológicas (imitación y lenguaje espontáneo) expliquen la discrepancia (Goldstein, Fabiano \& Iglesias, 2004). En este estudio la metátesis elicitada por imitación diferida desapareció con la edad, comportándose como los demás subprocesos. Por el contrario, en el trabajo de Goldstein et al. (2004) es asistemática en el habla espontánea de los niños ya que no disminuye con la edad. Por ello, su evolución no puede interpretarse con la misma lógica de los otros subprocesos.

Si bien es cierto que lo más característico es el uso de subprocesos estructurales, también se observó en el rango de 3 años una alta frecuencia de un subproceso sistémico relacionado con los fonemas líquidos (sustitución de líquidos entre sí). Este resultado concuerda con una inves- 
tigación efectuada en menores españoles de la misma edad en los que se advirtió una gran ocurrencia de tres subprocesos sistémicos. De ellos, dos afectan a los fonemas líquidos (ausencia de vibrante múltiple y conversión de la aproximante en líquida) (Bosch, 2004).

La sustitución de líquidos entre sí observada en este estudio consistió principalmente en el reemplazo de / / por / $/$ / o /l/. Una posible explicación del fenómeno se relaciona con la adquisición de esta clase de fonemas. Al respecto, se sabe que el fonema vibrante simple $(/ \mathrm{r} /) \mathrm{y}$ el lateral (///) presentan un $80 \%$ y un $90 \%$ de ocurrencia respectivamente entre los 3 y 4 años. En cambio, el fonema vibrante múltiple a los 3 años tiene un 50\% de aparición y a los 4 años un 70\% (Bosch, 1984), es decir, es de adquisición más tardía.

En relación a la variabilidad del rendimiento constatada en el trabajo, se advirtió que los participantes realizan diferentes subprocesos, aunque la mayoría de ellos presenta una frecuencia de uso menor al 50\%. Dicha diversidad corresponde principalmente a subprocesos de tipo estructural, lo que podría ser interpretado como una característica del desarrollo fonológico de esta etapa.

La variabilidad del uso de subprocesos se ha evidenciado en dos investigaciones realizadas con niños que tienen como lengua materna el español. En una de ellas, efectuada con portorriqueños de 3 y 4 años, se advirtió una gran diversidad en el uso de subprocesos sin que ninguno mostrara un alto porcentaje de ocurrencia (Goldstein \& Iglesias, 1996a). El otro estudio con niños españoles de 3 años estableció que solo 5 subprocesos, de un total de 27, alcanzan una frecuencia de uso sobre el $50 \%$ (estridencia de fricativas, ausencia de vibrante múltiple, conversión de la aproximante en líquida, reducción de grupo consonántico y simplificación de diptongo). El mismo estudio plantea que a los 4 años ningún subproceso supera un $50 \%$ de frecuencia. Los más comunes fueron estructurales y correspondían a simplificación de diptongo (47.1\%) y a reducción de grupo consonántico (43\%) (Bosch, 2004).

Los resultados hasta aquí comentados sugieren que los subprocesos estructurales, tanto en cantidad como en diversidad, son los preponderantes en el período del desarrollo fonológico estudiado. Esta evidencia supone que los niños están más centrados en adquirir la estructura de la palabra (estructura silábica y metría) que el sistema de oposiciones fonológicas.

El supuesto anterior puede basarse en la propuesta de la sensibilidad fonológica que plantea que los niños desarrollan la habilidad de manipular los sonidos del lenguaje oral. Esta habilidad se manifiesta inicialmente cuando los menores son capaces de detectar unidades fonológicas amplias (palabras o sílabas) y se advierte su mayor desarrollo cuando ellos pueden manipular los fonemas constitutivos de una palabra (Anthony, Lonigan, Burgess, Driscoll, Phillips \& Cantor, 2002). 
En cuanto a la evolución de la sensibilidad fonológica, se ha establecido que se adquiere entre los 2 y 5 años. Además, es posible observar una secuencia de adquisición de esta habilidad. Al respecto, se ha señalado que los niños en general manejan primero el nivel de la palabra, luego el de la sílaba, posteriormente el de los elementos constitutivos de ella y, por último, el del fonema. Por lo tanto, su progresión estaría determinada por la jerarquía que presentan las unidades lingüísticas dentro de la estructura de la palabra (Anthony, Lonigan, Driscoll, Phillips \& Burgess, 2003).

Basándose en el planteamiento previo, es posible postular que por la edad de los niños estudiados, ellos ya han adquirido la sensibilidad de la palabra. Por lo tanto, su foco de atención está en la sílaba, lo que significa que comienzan a tomar conciencia de que la palabra es una entidad divisible y que, por ello, está constituida por unidades menores que son las sílabas. Evidentemente, si los niños están en proceso de adquirir la habilidad para identificar y manipular la sílaba, ello implica que aún no manejan eficientemente ni la metría ni la estructura silábica, lo que se reflejaría en que sus producciones son simplificadas preponderantemente mediante subprocesos estructurales.

En relación a la influencia del género en el desarrollo fonológico, se observó que no existen diferencias significativas entre niños y niñas, lo que indica que este factor no es determinante en los desempeños fonológicos de los niños estudiados. Ello concuerda con investigaciones previas donde el género no incide en los PSF, los subprocesos y la producción de fonemas (Díez-Itza \& Martínez, 2003; Dioses et al., 2006). Además, es consistente con el desempeño de sujetos de 3 y 4 años hablantes de inglés en que ser niño o niña no influyó en su desarrollo fonológico, mientras que entre los 5.6 y los 6.11 años las niñas evidenciaron un mejor rendimiento (Dodd et al., 2003).

Tanto los resultados de este estudio como la evidencia obtenida en los otros trabajos citados sugieren que en niños menores de 5 años el desempeño fonológico no está influido por el género. Esta conclusión no es concordante con trabajos que señalan que la actuación lingüística es mejor en las niñas que en los varones (Bornstein, Haynes, Paiter \& Genevro, 2000; Dionne, Dale, Boivin \& Plomin, 2003).

Un hecho que podría incidir en los resultados obtenidos se relaciona con el tamaño muestral. Al respecto, existen antecedentes acerca de que las diferencias de género en el desempeño lingüístico se observan cuando se trata de estudios de casos o se utilizan muestras pequeñas (entre 12 y 47 sujetos participantes) con distribución similar entre niños y niñas (Plante, Schmithorst, Holland \& Byars, 2006). Dichas diferencias se anulan cuando el número de sujetos estudiados es mayor (Galián, Carranza \& Escudero, 2006). Por lo tanto, es importante que en 
las futuras investigaciones acerca de la influencia del género en el desarrollo fonológico se utilicen muestras más pequeñas.

En síntesis, los resultados de este trabajo permiten concluir que a los 3 y 4 años:

1. Los subprocesos de simplificación disminuyen significativamente entre los dos rangos etarios, en especial los estructurales.

2. Los subprocesos más característicos son preferentemente estructurales (Omisión de elementos átonos, Omisión de trabante y Asimilación idéntica) y solo a los 3 años existe sustituciones relacionadas con la adquisición de los fonemas líquidos.

3. El desarrollo fonológico se caracteriza por la gran variedad de subprocesos.

4. El género es una variable que no afecta el desarrollo fonológico en los niños estudiados.

\section{REFERENCIAS BIBLIOGRÁFICAS}

Acosta, V., León, S. \& Ramos, V. (1998). Dificultades del habla infantil: Un enfoque clínico. Málaga: Aljibe.

ADIMARK (2004). Mapa socioeconómico de Chile. Nivel socioeconómico de los hogares del país basado en datos del Censo [en línea]. Disponible en: http://www.adimarkgfk.com/medios/estudios/Mapa_Socioeconomico_de_Chile.pdf

Anthony, J., Lonigan, C., Driscoll, K., Phillips, B. \& Burgess, S. (2003). Phonological sensitivity: A quasi-parallel progression of word structure units and cognitive operations. Reading Research Quarterly, 38(4), 470-487.

Anthony, J., Lonigan, C., Burgess, S., Driscoll, K., Phillips, B. \& Cantor, B. (2002). Structure of preschool phonological sensitivity: Overlapping sensitivity to rhyme, words, syllables, and phonemes. Journal of experimental child psychology, 82(1), 65-92.

Bauer, D., Goldfield, B. \& Reznick, J. (2002). Alternative approaches to analyzing individual differences in the rate of early vocabulary development. Applied Psycholinguistics, 23, 313-335.

Bassano, D., Maillonson, I. \& Eme, E. (1998). Developmental changes and variability in early lexicon: A study of French children's naturalistic production. Journal of Child language, 25, 493-531.

Belinchón, M., Igoa, J. \& Rivière, A. (2000). Psicología del lenguaje. Investigación y teoría. Madrid: Ed. Trotta.

Bornstein, M., Haynes, O., Painter, K. \& Genevro, J. (2000). Child Language with mother and with stranger at home and in the laboratory: A methodological study. Journal of Child Language, 27,407-420. 
Bosch, L. (1984). El desarrollo fonológico infantil: Una prueba para su evaluación. En M. Siguán (Ed.), Estudios sobre psicología del lenguaje infantil (pp. 33-58). Madrid: Pirámide.

Bosch, L. (2003). Trastornos del desarrollo fonético y fonológico. En M. Puyuelo \& J. A. Rondal (Eds.), Manual de desarrollo y alteraciones del lenguaje (pp.189-204). Barcelona: Masson.

Bosch, L. (2004). Evaluación fonológica del habla infantil. Barcelona: Masson.

Burman, D., Bitan, T. \& Booth, J. (2008). Sex differences in neural processing of language among children. Neuropsychologia, 46, 1349-1362.

Carter, A. \& Gerken, L. (2003). Similarities in weak syllable omission between children with specific language impairment and normally developing language: A preliminary report. Journal of Communication Disorders, 36(2), 165-179.

Clemente, R. (1996). Desarrollo del lenguaje. Barcelona: Octaedro.

Díez-Itza, E. \& Martínez, V. (2003). Procesos de metátesis en el desarrollo fonológico de niños de 3 a 6 años. Psichotema, 15(2), 285-291.

D’Introno, F., Del Teso, E. \& Weston, R. (1995). Fonética y fonología española. Madrid: Cátedra.

Dionne, G., Dale, P., Boivin, M. \& Plomin, R. (2003). Genetic evidence for bidirectional effect of early lexical and grammatical development. Child Development, 74, 394-412.

Dioses, A., García, L., Matlinares, M., Cuzcano, A., Panca, N., Quiroz, J., Fernández, C. \& Castillo, J. (2006). Análisis psicolingüístico del desarrollo fonético-fonológico de alumnos pre-escolares de Lima Metropolitana. Revista de Investigación en Psicología, 9(2), 9-32.

Dodd, B., Holm, A., Hua, Z. \& Crosbie, S. (2003). Phonological development: A normative study of British English children. Clinical Linguistics \& Phonetics, 17(8), 617-643.

D’ Odorico, L., Carubbi, S., Salerni, N. \& Calvo, V. (2001). Vocabulary development in italian children: A longitudinal evaluation of quantitative and qualitative aspects. Journal of Child Language, 28, 351-372.

Galeote, M. (2002). Adquisición del lenguaje. Madrid: Pirámide.

Galián, M., Carranza, J. \& Escudero, A. (2006). Variación estilística en la adquisición del léxico inicial. Anales de Psicología, 22(1), 98-104.

Garayzábal, E. (2006). ¿Rasgos distintivos o dislalia? La Lingüística y la Logopedia: fronteras y disciplinarias y disciplinas complementarias. En E. Garayzábal (Ed.), Lingüística Clínica y Logopedia (pp.21-79). Madrid: A. Machado Libros.

Goldstein, B. \& Iglesias, A. (1996a). Phonological patterns in normally developing Spanishspeaking 3- and 4- years-old of Puerto Rican descent. Language, Speech, and Hearing Services in Schools, 27, 82-90. 
Goldstein, B. \& Iglesias, A. (1996b). Phonological patters in Puerto Rican Spanish-speaking children with phonological disorders. Journal Communication Disorders, 29, 367-387.

Goldstein, B., Fabiano, L. \& Iglesias, A. (2004). Spontaneous and imitated productions in Spanish- speaking children with phonological disorders. Language, Speech and Hearing Services in Schools, 35, 5-15.

ICCOM (2005). Descripción básica de los niveles sociales [en línea]. Disponible en: http: //www. iccom.cl

Ingram, D. (1983). Trastornos fonológicos en el niño. Barcelona: Médica y Técnica.

Law, J. (1992). The early identification of language impairment in children. London: Chapman \& Hall.

Martínez, V., Diez-Itza, E. \& Miranda, M. (2004). Detección de alteraciones fonológicas a partir de índices de error en el habla espontánea. Actas del XXIV. Congreso Internacional AELFA, Madrid, 449-464.

Murray, A., Johnson, J. \& Peters, J. (1990). Fine-tuning of utterance length to preverbal infants: Effects on later language development. Journal of Child Language, 14, 511-525.

Navarro Tomás, T. (1966). Estudios de fonología española. Nueva York: Las Américas Publishing Company.

Oropeza, M. (2001). Adquisición de la estructura silábica del español en niños de dos a seis años. Colección Pedagógica Universitaria, 36, 1-13.

Pavez, M., Maggiolo. M. \& Coloma, C. (2008). Test para evaluar procesos de simplificación fonológica. Versión revisada. TEPROSIF R. Santiago de Chile: Ediciones Universidad Católica.

Plante, E., Schmithorst, V., Holland, S., \& Byars, A. (2006). Sex differences in the activation of language cortex during childhood. Neuropsychologia, 44, 1210-1221.

Roulstone, S., Loader, S. \& Northstone, K. (2002). Descriptive data from the Avon longitudinal study of parents and children. Early Child Development and Care, 22, 259-268.

Serra, M., Serrat, E., Solé, R., Bel, A. \& Aparici, M. (2000). La adquisición del lenguaje. Barcelona: Ariel Psicología.

Stampe, D. (1969). The acquisition of phonetic representation. Papers from the Fifth Regional Meeting of the Chicago Linguistic Society, Chicago: Chicago Linguistic Society, 433444.

Storti, P. (2002). Estudio descriptivo sobre los procesos fonológicos de simplificación en niños de 2 años a 5 años. Tesis de Licenciatura, Universidad Nacional del Rosario, Rosario, Argentina. 